\title{
DIE SÄKULARISIERUNG DER ITALIENISCHEN GESELLSCHAFT IM SPIEgEL DER BANKENNAMEN
}

\author{
FIORENZA FisCHER \\ Wirtschaftsuniversität Wien, Austria
}

\section{The secularisation of Italian society mirrored in bank names}

\begin{abstract}
This study deals with Italian bank names and their structural changes in the context of Italian society. Bank names, as names of organisations, are the result of an intentional naming process. Such names are consciously created and are used to identify, distinguish and orient in economic, cultural, religious and social life. From an onomastic point of view, they reflect not only the history of language, but also in a profound way the historical events and cultural transformation of a country. In this sense, this study shows that bank names mirror the Italians' changing attitude towards church and religion. Based on selected examples of bank names, the paper aims at illustrating concisely the development of the secularisation of Italian society from the Late Middle Ages until today.
\end{abstract}

Keywords: bank names, economic history, secularisation, Italy.

\section{Einleitung}

Bankennamen sind vom onomastischen Standpunk nicht eindeutig klassifizierbar. Das Problem liegt nicht darin, dass heterogeneObjekte zu benennenwären ${ }^{1}$, über denBegriff, Bank' herrschtim Allgemein Konsens ( vgl.Gabler, Wirtschaftslexikon), auch wennman Bankenin mehreren Typologien unterscheiden kann (Kommerzbanken, Geschäftsbanken, Investmentbanken, Zentralbanken u.s.w.). Nach dem derzeit geltenden Klassifikationsansatz der Onomastik (Nübling/Fahlbusch/Heuser 2012) entsprechen Bankennamenje nachdem, ob die Banken privatwirtschaftlich und gewinnorientiert oder gemeinnützlich operieren,entweder der Definition von Unternehmensnamen (vgl. Falbusch 2012: 277) oder der Definition von Institutionsnamen (vgl. Vasil'eva 2004: 605-621). Für Bankenamen scheint eine Abgrenzung zwischen den zwei Subklassen nicht leicht nachvollziehbarzu sein, zumal eine und dieselbe Bank kann in unterschiedlichen wirtschaftshistorischen Phasengeführt worden sein, wie eine öffentliche Institutionim Dienste des gemeinsamen Interesse, oder wie ein Unternehmen mit dem Ziel, Profit zu erwirtschaften. Um dieses Problem umzugehen, wird hier die übergeordnete Klasse der Oganisationsnamen(vgl. Fischer in: Mautner/Rainer 2017:

1 Vgl. Die Problematik der Klassifizierung in Namenklassen in Nübling, D./Fahlbusch, F./ Heuser, R.(2012): 2086-297.f 
544-550 $)^{2}$ herangezogen. Organisationsnamen sindvon Menschen bewusst kreierte Benennungen für von Menschen geschaffene Einrichtungen. Organisationsnamen dienen zur Identifizierung, Unterscheidung und Orientierung in Wirtschafts-, Kultur- und Gesellschaftsleben und in diesem Sinne widerspiegeln sie die Entwicklungsprozesse und den Wertewandel einer Sprechergemeinschaft.

Diese Untersuchung versucht in diesem Sinne zu zeigen, wie in Laufe der Geschichtein Italien die Bankennamendie Veränderung der italienischen Gesellschaft in Bezug auf Religiosität widerspiegeln. Dabei wird hier kein exhaustiver, quantitativer Forschungsansatz angewendet, es werden viel mehr anhand von typischen Beispielen die allgemeinen Linien der Evolution der Bankennamen im Verhältnis zur religiösen Einstellung der Sprechergemeinschaft.

\section{Forschungshintergrund}

Die jetzige Forschung schließt an zwei anderen Untersuchungen über Bankennamen: Fischer (2008), Fischer und Wochele (2012). Einige der aus diesen Arbeiten gewonnenen Erkenntnisse sind auch hier von Wichtigkeit, daher wird nun kurz darauf eingegangen. Die erste Arbeit zielte darauf ab herauszufinden, wie sich die Fusionierungsprozesse des Zeitraums 1990-2006 auf die daraus resultierenden Bankennamen auswirkten. Sie brachte die wichtige Erkenntnis, dass im Kreditsektor bei der Wahl der Namen für Banken eine konservative Haltung herrscht. Es sei nebenbei erwähnt, dass der Finanzfachterminus 'Kredit' vom lat. credere = glauben/vertrauen stammt ( $v g l$. Fischer 2015). Im Kreditsektor sind Solidität, Zuverlässigkeit und Beständigkeit relevante Faktoren. Sie manifestieren sich auf der Ebene der Sprache unter anderem auch in der Beständigkeit der Bankennamen. Ein klares Beispiel in dieser Hinsicht bietet die italienische Bank Monte dei Paschi di Siena. Sie gilt heutzutage als die älteste, existierende Bank der Welt, sie wurde im Jahr 1472 gegründet und führt nach einem halben Jahrtausend immer noch den gleichen unveränderten Namen. Auf die Bedeutung dieses Namens wird später eingegangen.

Kontinuität und Stabilität bilden also die Basis des Vertrauens für Kunden. Der Experte für Bankmarketing Maier bekräftigt die Wichtigkeit des Vertrauens. Er schreibt (1999: 32): „Grundsätzlich spielt das Vertrauen eine zentrale Rolle bei Bankleistungen. In der Regel muss der Kunde bei Inanspruchnahme von Bankleistungen dem Kreditinstitut Vertrauen entgegen bringen“"3. In der Folge wird also untersucht, wie bei Bankennamen Vertrauen und religiöser Glaube eng verknüpft sind.

Die zweiterwähnte Forschungsarbeit behandelte das Thema der nationalen und

\footnotetext{
2 Aus onomastischer Sicht ist der Sammelbegriff Organisationsnamen eine pragmatische Lösung mit dem Zweck, die zwei Klassen der Institutionsnamen und Unternehmensnamen auf einen gemeinsamen Nenner zu bringen.

3 Ein Bankkunde, der heute sein Geld bei einer Bank einlegt mit der Aussicht, in der Zukunft den gleichen Betrag plus einem Zinsertrag zu bekommen, handelt im Vertrauen und emotional mit „high involvement", weil ein sicheres Verfügen über sein Erspartes direkten Einfluss auf sein Leben hat.
} 
europäischen Identität bei italienischen, ungarischen und rumänischen Bankennamen in der Gegenwart. Bei dieser Arbeit stellte sich heraus, dass die typischen Elemente, die bei der Benennung einer Bank häufig vorkommen, folgende sind:

1) Banktypologie

2) Regionale Zuständigkeit

3) Wirtschaftssektor und

4) Anthroponyme

Bei der Analyse dieser Elemente stellte sich außerdem heraus, dass die Wahl eines Bankennamen ein intendierterund selbstgesteuerter Akt ist. Die Wahl des Namens ermöglicht einem Kreditinstitut sein Selbstbild zu präsentieren: Diese Form der Selbstdarstellung hinsichtlich Leistungsangebot, sozialer Werte und Arbeitsweise bildet ein Identifikationsangebot für/anKunden. Das gilt heutzutage noch genauso wie im Spätmittelalter. Schon damals konnte der fromme Sparer sein erspartes Geld bei dem Sacro Monte dei Poveri di Perugia und der reiche Händler seines hingegen bei dem Banco de' Bardi di Firenze oder bei der Gran Tavola de’ Bonsignori di Siena einlegen.

Eine dritte wichtige Erkenntnis entsprang aus dem Vergleich zwischen ungarischen, rumänischen und italienischen Bankennamen. Es stellte sich heraus, dass nur die letzteren Hagionyme oder andere religiös motivierte Bildungselemente enthalten. Ein Vergleich mit Bankennamen aus dem deutschen Sprachraum bestätigte die Beobachtung, dass dieses ein Spezifikum Italiens zu sein scheint. Auf der Grundlage dieser Entdeckung basiert die heutige Studie.

\section{Forschungsfragen}

Die Forschungsfragen, auf die in dieser Untersuchung versucht wird, Antwort zu finden, sind:

1) Abwann tauchen Hagionyme oderandere religiös motivierte Bildungselemente bei italienischen Bankennamen auf?

2) Welche wirtschaftshistorischen Rahmenbedingungen liegen dieser sprachlichen Erscheinung zu Grunde?

3) Wie entwickelt sich im Verlauf der Geschichte diese spezifisch italienische Besonderheit der religiös konnotierten Bankennamen?

\section{Heilig und Profan. Grundlegende Überlegungen}

In einer ersten Annäherung an das Thema „Heilig vs./und Profan in der Onomastik“ wird für eine Definition von „Hagionym“auf Krüger (2004) ${ }^{4}$; Greule (2016) und Dräger/Fahlbusch/Nübling (2016) verwiesen. Im Zusammenhang mit Banken bzw. Geldhäuser scheint es zunächst logisch, dass das zu benennende Forschungsobjekt dem Bereich des Weltlichen zuzuschreiben ist. Geld als Inbegriff des

\footnotetext{
4 Krüger, Dietlind (2004): Textlinguistische Methoden der Namenforschung. In: Brendler, Andrea/Brendler, Silvio (Hgg.): Namenarten und ihre Erforschung. Ein Lehrbuch für das Studium der Onomastik. Hamburg, 123-152.
} 
'Materiellen' scheint logischerweise der Domäne des Diesseits anzugehören. Doch ein Blick zurück in die Geschichte des Geldes verrät dem Sprachforscher, dass schon seit der Antike eine enge Verbindung zwischen Tempeln und Geld bestand, so verdeutlicht z.B. die Etymologie des Wortes <Moneta>/Münze, dass im antiken Rom ursprünglich Münzen im Tempel der Göttin Juno Moneta geprägt wurden. Auch das germanische Wort <Geld>, ursprünglich „Opfergabe“, hatte also eine religiöse Bedeutung ( vgl. Fischer 2015).

In der Antike waren Tempel unantastbare, sichere Orte, in denen nicht nur reichlich Opfergaben flossen sondern auch Thesauri bzw. materielle Werte sicher aufbewahrt werden konnten. Diese Verbindung zwischen Geld und Tempel bestand schon in Mesopotamien (sogenannte „Tempelwirtschaft") bei den Sumeren und in der griechisch-römischen Kultur. Sie wird bezeugt auch in der Hebräischen Kultur, wie die Stelle des Evangeliums verdeutlicht, in der Jesus die Händler vom Tempel vertreibt und die Tische der Geldwechsler umstößt $\mathrm{t}^{5}$. Die Geldwechsler (lat. auch nummularii oder argentarii genannt) hatten damals die Aufgabe, den Wert der Münzen zu schätzen und Währungen zu wechseln, aber sie nahmen auch Geldeinlagen gegen Zins und boten zu höherem Zins Kredit an. Mit anderen Worten: Sie waren die Bankiers der Antike. Nach der Christianisierung wurde den Christen im Mittelalter von der Kirche verboten, Geld zu verleihen und dafür Zins zu lukrieren. Dies würde als lat. usura (Wucher) verpönt und als Sünde bzw. Häresie gewertet. Diese profane Tätigkeit blieb in der Folge den Juden vorbehalten und sie begannen sich allmählich darin zu spezialisieren, zumal ihnen verboten war, andere Berufe auszuüben.

\section{Entstehung und Benennung der Banken in Italien im Spätmittelalter}

Aus der mittelalterlichen Perspektive war Geld deutlich etwas Profanes. Geld wurde als steriles Gut gesehen (pecunia pecuniam non parit) und das Einheben von Zinsen als wider der Natur gewertet. Wirtschaftshistorisch veränderten sich aber gegen Ende des Mittelalters die Rahmenbedingungen. Nach der Jahrtausendwende entwickelten sich in Nord- und Mittelitalien die Stadtstaaten. Die Handwerker - organisiert in Zünften - erzielten große Fortschritte in der Warenerzeugung und folgerichtig begann der Handel im Mittelmeer sowie in Richtung Flandern, England und Frankreich zu florieren. Es entstand somit ein steigender Bedarf nach Geldmenge und Bankleistungen wie Auszahlungen, Überweisungen und Wechsel. Italien, genauer gesagt die Toskana, wurde - wie allgemein bekannt - die Wiege und der Geburtsort der Banken. Erfolgreiche Händler in der Toskana begannen das Vermögen, das sie durch merkantile Tätigkeit angehäuft hatten, in Bankoperationen einzusetzen. Sie konnten weit gespannte, effiziente Netze von Filialen, Agenturen und Lagern nutzen, um Bank- und Finanzgeschäfte abzuwickeln. Die „compagnie mercantili“ verwandelten sich allmählich in „compagnie di banco“.

In Florenz entstanden: Banco de' Bardi, Banco Acciaiuoli, Banco Peruzzi, in Siena

5 Mr 11:11, 15-19; Lu 19:45-48; Gv 2:13-22, Mt 21, 12-17. 
Gran Tavola de Bonsignori und später derBanco de' Medici und die Banken der Familien: Pazzi, Rapondi, Guinigi, Strozzi, Chigi.

Auch die Biografie des Giovanni Boccaccio bietet einen Einblick in die Netzstruktur dieser früheren Banken. Sein Vater Boccaccino war für die Familie Bardi „agente di cambio“ in Paris und nahm später den vierzehnjährigen Sohn Giovanni nach Neapel, wo der Dichter ebenfalls den väterlichen Beruf bei dem Banco de Bardi di Napolihätte erlernen sollen.

Neben den „banchi privati“ entstanden in Italien zwischen dem dreizehnten und fünfzehnten Jahrhundert auch folgende „banchi pubblici“: Casa delle Compere e dei Banchi di San Giorgio (Genova), Tavola Pecuniaria di Palermo,Tavola Pecuniaria di Messina, Monte dei Paschi di Siena, Monte Comune di Firenze, Banco della Piazza di Rialto (Venezia).

Die banchi pubblici erledigten nicht nur die üblichen Depot-, Giro- und Diskontoperationen wie jede andere Bank, sie führten auch Zahlungen im Auftrag der öffentlichen Verwaltung aus. Die erste Bank mit einer Funktion in der öffentlichen Verwaltung war die Casa delle Compere di Genova. „Le compere“ waren die öffentlichen Ausgaben der Seerepublik Genua, daher der Name "Casa delle compere" für die ausführende Bank. Wenn man aus semantischer Sicht die Bankennamen dieser zwei Typologien von Banken - „banchi privati“ und „banchi pubblici“ - unter die Lupe nimmt, kann man feststellen, dass die Bildungselemente der Namen folgende sind:

1.) Banktypologie:

Tavola Pecuniaria, Gran Tavola, Monte,Casa delle Compere, Banco

2.) Geografische Bezeichnung:

(dei Paschi) di Siena, di Firenze, di Messina, di Palermo, di Piazza di Rialto, di S. Giorgio

(In diesemletzten Fall ist der Bankenname nicht intrinsisch religiös motiviert. S. Giorgio ist hier nur einoikodomonymischer Hinweis für Palazzo di S. Giorgio, den Palast vor dem die Tische der Geldwechsler aufgestellt wurden).

3.) Anthroponym: Familiennamen des Bankgründers/Inhabers.

Bardi, Acciaiuoli, Pazzi, Strozzi, Bonsignori, Chigi

4.) Wirtschaftssektor:

Paschi (Weidewirtschaft)

Insgesamt können all die Bildungselemente der Benennungen der banchi privati und der banchi pubblici semantisch als weltlich und komplett profan eingestuft werden. Die in den Namenbildungen vorkommenden Namenvon Familien, Ortschaften, Objekte gehören zweifelsohne der Domäne des Diesseits an.

\section{Der Einfluss der Franziskaner auf Gesellschaft und Banken im Spätmittelalter}

Parallel zu den geschilderten wirtschaftshistorischen Entwicklungen ereigneten sich in der italienischen Gesellschaft tiefgreifende Veränderungen, die großen Einfluss auf die Typologie und auch auf die Namen der Banken haben werden. Gegen 
Ende des Mittelalters findet unter dem Einfluss der Franziskaner eine Art religiöses Erwachen statt. Der ökonomische Hintergrund für das Umdenken ist von einer Entwicklunggeprägt, in der der Reichtum in Summe stark steigt - und das Vermögen der oberen Schichten im Besonderen - während die Randschichten der städtischen Bevölkerung gleichzeitig von zunehmender Verarmung betroffen sind. In Not geratene kleine Handwerker oder Händler sind um zu überleben auf kleine Kredite angewiesen. Die großen „banchi privati“ gewähren aber keine kleinen Kredite mehr. Sie sind inzwischen wichtige Kreditgeber der Päpste oder französischer und englischer Monarchen geworden. Das Marktsegment des kleinen Konsumkredits ist fast zur Gänze in den Händen der Juden. Die sogenannten „ebrei condotti“ verfügen über eine offizielle "condotta", eine Genehmigung der Staatsverwaltung, die sie legal befähigt, gewerblich Geldgeschäfte zu betreiben ${ }^{6}$. Die von den banchi ebraici praktizierten Zinsen liegen allerdings im Durchschnitt bei 15- 20\% und erreichen sogar 30\% für die ländliche Bevölkerung - ein zu hohes Niveau für die Verarmten, die kleine Kredite benötigen und auf diese Weise tiefer in der Armutsspirale versinken. Angesichts des Elends beginnen viele Bürger sich zu karitativen Zwecken in confraternitates, congregationes, consortia, sodalitia, gilde, fraglie und compagnie zu vereinen.

Sie gründen scholae, Lazarette und Spitäler um Waisen, alten und kranken Menschen zu helfen. Diese compagnie - gebildet von Laien und Geistlichen - werden später im 16. und 17. Jahrhundert sehr einflussreich werden und bei Bankgründungen eine wichtige Rolle spielen.

Die desolaten sozialen Umstände bewirken aber auch innerhalb des Franziskanerordens ein Umdenken. Der Wucher als Ursache der Armut wird von ihnen immer noch an den Pranger gestellt. Sie beginnen aber allmählich zu begreifen, dass nicht nur Almosen sondern Mitteln zur Selbsthilfe an die sogenannten „pauperes pinguiores" gegen Armut nachhaltiger wirken würde ${ }^{7}$. Der heilige Thomas von Aquin legt theologisch die Basis für eine erneuerte Theorie des gerechten Preises und des Geldes und der Franziskaner BernardinusvonSiena schafft mit seinem „Tractatus de contractibus et usuriis" die Grundlage für eine Reform der christlichen Wirtschaftsethik. Nach dem neuen Denkansatz ist es ein Akt der Barmherzigkeit, wenn Christen anderen in Not geratenen Christen mit karitativ gesammelten Geldern helfen. Auch wenn eben diese Gelder gegen Pfand bzw. gegen symbolische oder sehr moderate Zinsen geliehen werden. Das Prinzip einer „christlichen Bank“ wird so eingeführt und das Bankmodell des Mons Pietatis (Pfandleihe der Barmherzigkeit) wird von den Franziskanern propagiert. Barnaba Manassei, Fortunato Coppoli, Michele Carcano - jeweils dem Orden der Franziskaner angehörig - verbreiten mit ihren Predigen und Schriften die neue soziale und wirtschaftliche Ethik zuerst in Umbrien. Bernardinus von Feltre, ein begnadeter Prediger, der jedoch oft auch antijüdische Töne anschlägt, macht es zu seiner Lebensaufgabe, Monti di Pietà zu gründen. Die erste Gründung in diesem Sinne findet

Vgl. Todeschini 1989 und 2016.

Vgl. Muzzarelli 2001. 
in Perugia im Jahr 1462 statt $^{8}$. Der vollständige Bankname lautet: Monte de la Pietà et de la Virgine Maria matre de misericordia. Die Bankennamen der ersten christlichen Bankinstitutionen sehen folgenderweise aus:

Monte de la Pietà et de la Virgine Maria matre de misericordia o ancheMonte dei Poveri di Perugia

Monte de la Pietà et de la Virgine Maria matre de misericordia (Assisi)

Monte di Christo di Orvieto (1463)

Monte pio di Macerata

Monte di santa Maria della Pietà (Jesi 1472)

Monte dei Meriti di Ancona

Monte di Pietà di Parma

Santo Monte della Pietà in Faenza

Monte della Pietà di Vicenza (1486)

Sacro Monte della Pietà di Reggio Emilia (1494)

Monte della Pietà o del Soccorso dei poveri della città Milano, (1497)

Santa Casa della Pietà o Monte di Pietà di Verona

Santo Monte di Pietà di Trento (1523)

Pio Monte della Misericordia di Napoli (1602)

Muzzarelli (2001) zählt im Jahr 1515 bereits 135 Monti di Pietà.

Die sprachliche Analyse der Bildungselemente dieser Bankbenennungen zeigt folgendes:

1.) Typologie der Bank: lat. mons, it. monte (Berg, Haufen, Anhäufung, Summe)

Dieser Bestandteil wird erst ab jetzt neulich von Adjektiven wie sacro, santo, pio (heilig/fromm) als Hinweis aufdie sakrale Natur der Bankinstitution begleitet.

2.) Geografische Angabe: Name der Stadt: Perugia, Ancona, Orvieto, Forli etc.

3.) Tätigkeitsfeld: Zweck bzw. modus operandi der Bank: pietà, misericordia, soccorso

Diese sind Tugenden der christlichen Lehre.

4.) Target group: i poveri, i poveri della città di Milano

Zum Begriff lat. mons it. Monte muss man anmerken, dass dieser Ausdruck schon viel früher in der Sprachverwendung im Umlauf war. Er war aber nicht religiös konnotiert ${ }^{9}$. Die metaphorische Übertragung aus dem Spenderbereich $<$ Berg $>$ bezog sich ursprünglich auf eine physische Form, auf ein Volumen oder eine Menge materieller

8 Muzzarelli (2001: 18) zitiert Originalquellen: „Hic mons pietatis primus in orbe fuit osservò un consigliere Perugino. 'Qui mons jure mons perusinus dicitur, cum ab ipse civitate primitus erectus sit, tamquam candelabrum lucen ferens, modo ab omnibus fidelibus merito imitandum." [consilium doctorum perusiae edito in pro mons pietatis consilia, Venezia, Giovanni Tacuino, 1495-97].

9 Schon in der Kultur der Agrargesellschaft waren in Italien monti frumentari (Getreideberg/Getreidebank) bekannt. Bauern konnten vor der Saat einen Behälter gestrichen voll mit Getreide erhalten und mussten nach der Ernte den gleichen Behälter, voll angehäuft retournieren. 
Güter. Das Wort überlebt auch später im profanen Bereich mit ebendieser Bedeutung (monte della lana, della canapa, delle castagne) oder im übertragenen Sinn von „Summe“ in der Neuzeit wie folgende Beispiele beweisen ${ }^{10}$ :

Monte dell'Utilità (Venezia)

Monte delle Doti (Firenze)

Monte dei Matrimoni (Bologna)

Monte dei Maritaggi (Napoli)

Monte dei gentiluomini cortigiani (Napoli)

Das Wort monte existiert heutzutage noch immer in der italienischen Fachterminologie der Versicherungen und der Börse z. B. in Termini wie: monte-titoli, monte-cedole, monte-premi.

Man soll nunauf den Bedeutungswandel, den der Begriff Monte durch die Predigtender Franziskanererfährt, eingehen. Muzzarelli (2001: 87-90) analysiert den Begriff < Mons> (Berg) im Kontext der christlichen Kultur: Der „Mons Dei, Mons Pinguis" (der schon in der Heiligen Schrift zu finden ist) ist ein Gottesberg, weil von Gott gewollt. Dieser Berg verbindet Erde und Himmel. In der Auffassung der Franziskaner ermöglicht der Monte di Pietà, Christen durch Almosen, Spenden und durch das Einlegen ihrer Ersparnisse, Barmherzigkeit gegenüber anderen Christen zu praktizieren und so ihre Seele zu retten.

Diese neue Perspektive ermöglicht es, Sünde und Nutzung des Geldes voneinander zu trennen und erlaubt Christen, ihr Geld gegen moderaten Zins dank dem Monte di Pietà an Arme zu verleihen. Somit wird Geld nicht gehortet sondern in Umlauf gebracht. Dies entspricht ganz genau den Bedürfnissen der Wirtschaft in dieser Phase vonGeldmengenknappheit und gerade deswegen erweist sich das neue Bankmodell als extrem erfolgreich.

Imneuentstandenen kulturellen Klima scheint sich die Verflechtung zwischen Bank und Religion in der kollektiven Denkweise zu verbreiten und darin tief zu verankern. Auf sprachlicher Ebene manifestiert sich dieser neue Umstand dadurch, dass neue Bankennamen neue Strukturen aufweisen ${ }^{11}$ :

Von Hagionymen geprägte Benennungen für Monti di Pietà waren offensichtlich so verbreitet und populär geworden, dass sie für die Sprechergemeinschaft üblich und vertraut geworden waren. Namenstrukturen mit religiösen Bildungselementen wurden somit als Vorbilder empfunden und dienten als Muster für weitere Banknamenschöpfungen.

Im 17. Jahrhundert kann man eindeutig eine intensive Verwendung von Hagionymen in Bankennamen feststellen, wie folgende Beispiele zeigen:

10 Siehe dazu Gugnoni Gabriele $(2010,14)$

11 Hier eine Beschreibung der inneren Räumlichkeiten des Sacro Monte di Pietà di Bologna:" [...] Uno dei locali del Monte di Pietà, nel quale vi era il Monte di S. Antonio abbate, che riceveva in deposito e pegno canepa; quello di S. Domenico, gioie, oro, argento ed arnesi; e quello di S. Francesco, per soli arnesi ad uso de' mestieri o delle arti manuali [... ]“ (Fonte: N.1627,1628 Dalle „Cose Notabili“ di Giuseppe Guidicini). 
Banco di San Paolo (Turin)

Banco di Sant'Ambrogio (Mailand)

Banco di Santo Spirito (Roma)

Banco di San Marco (Venezia)

Banco di San Giorgio (Genova)

Die endgültig etablierte Verflechtung zwischen Religion und Bank erscheint in voller Deutlichkeit, wann auch Namen von „banchi pubblici“ religiös konnotiert werden. Es wird hier exemplarisch die Auflistung von acht Geldhäusern angeführt, dokumentiert im Archivio Storicoder Fondazione Banco di Napoli:

All'inizio del 1500, Napoli era la seconda città europea dopo Parigi; il suo porto commerciale era uno dei maggiori del Mediterraneo. Tra il 1500 ed il 1600 sorsero dunque gli otto banchi pubblici napoletani:

1. Banco della Pietà (1539-1808);

2. Banco dei Poveri (1563-1808);

3. Banco dell'Annunziata (1587-1702);

4. Banco di Santa Maria del Popolo (1589-1808);

5. Banco dello Spirito Santo (1590-1808);

6. Banco di S.Eligio (1592-1808);

7. Banco di S.Giacomo e Vittoria (1597-1809);

8. Banco del Santissimo Salvatore (1640-1808).

Bis auf das Wort Banco, das auf die Banktypologie hinweist, alle anderen Banknamenbestandteile (Barmherzigkeit, Heiligennamen) der stammen aus der Domäne der christlichen Religion.

Die religiöse Konnotation, die im 15. Jahrhundert nur bei den Namen der von den Franziskanern gegründeten Monti di Pietà zu finden war, verbreitet sich später auf weite Teile des Banksektors und ist sogar bei den Namen der „banchi pubblici“ nicht mehr wegzudenken.

\section{Napoleon und der Einfluss der Aufklärung auf Gesellschaft und Bankennamen}

Der Einfluss der Religion und dieFülle an Hagionymen in Bankennamen erlebt in der letzten Dekade des 18. Jahrhundertseinen Einbruch: Napoleon besetzt mit seinen Truppen TeileNord- und Mittelitaliens, viele Klöster werden aufgelöst und die vollen Kassen der Monti diPietà werden als Kriegsbeute geplündert. Dieses Ereignis geht in die Italienische Geschichte mit demNamen „Spoliazione Napoleonica“ ein. Die Enteignung durch Napoleon stellt für dieMonti di Pietà eine dramatische Zäsur dar. Viele Monti können sich - nachdem sie ihre ganzen Reserven verloren haben nicht mehr erholen. Nur wenige werden den früheren Glanz wieder erreichen. Die Auswirkung auf der Ebene der Bankennamen bleibt nicht aus, wie das markanteste Beispiel der Umbenennung der mailänder Bank vor Augen führt:

Banco di Sant'Ambrogio-> Monte di Santa Teresa->Monte Napoleone->Imperial Regio Monte Lombardo Veneto 
In diesem emblematischen Fall wirkt sich die Säkularisierung der Gesellschaft in aller Deutlichkeit auf die Benennung der Bank aus.

Die napoleonische Phase bringt die katholische konservative Gesellschaft Italiens mit den Ideen der Aufklärung in Kontakt. Der rationale, weltliche Denkansatz aus Frankreich fasst bei den italienischen intellektuellen Eliten langsam Fuß. Nach der Restauration 1815 kommt der Banksektor zum Teil wieder zur Ruhe, in der Lombardei jedoch führt die Habsburgische Verwaltung nicht nur die Grundschulbildung für alleBürger ein, sondern auch ein innovatives Bankmodell, welches in Frankreich und Deutschland schon verbreitet war: die Sparkasse. In ihrer Konzeption war die Sparkasse ein Produkt des philanthropischen Denkansatzes der Aufklärung. Die Bürger sollen zur Kultur des Sparens und des Vorsorgens erzogen werden.

Dieses neue Bankmodell - nämlich die Sparkasse - wird die Landschaft der Bankennamen in Italien radikal erneuern und endgültig säkularisieren.

Vom wirtschaftshistorischen Standpunkt aus kann man mit Sicherheit sagen, dass zu der Zeit das Bankmodell der Sparkasse dierichtige Antwort auf die geänderten Verhältnisse der Wirtschaftswelt am Vorabend der Industrialisierungwar. Die erste Sparkasse wurde in Venedig 1822 errichtet.

Es folgten die Cassa di Risparmio di Padova e Rovigo (1822) und die Cassa di Risparmio Delle Provincie Lombarde (1823).

\section{Die Gegenwart: Bankennamen und Gesellschaft im heutigen Italien}

Die Benennungen der Sparkassen verdeutlichen in ihrer klaren Struktur die stark veränderten historischen und kulturellenVerhältnisse der italienischen Gesellschaft. Die Ortsangabe bekommt in den neuen onymischen Strukturen eine größere Relevanz. Es werden- nebenbei bemerkt - immer mehr Toponyme von kleineren Städten oder gar Dörfern benutzt. Dieser Umstand ist Ausdruck des weit verzweigten Netzes bis in ländliche Gebiete, und gleichzeitigzeigt er auch wie tief verwurzelt diese Kreditinstitute im lokalen Wirtschaftsleben sind, wie folgende Beispiele beweisen:

Cassa di Risparmio di Carrara

Cassa di Risparmio di Cesena

Cassa di Risparmio di Chieti

Cassa di Risparmio di Città di Castello

Cassa di Risparmio di Civitavecchia

Cassa di Risparmio di Cortona

Cassa di Risparmio di Cuneo

Cassa di Risparmio di Fabriano e Cupramontana

Aus onomastischer Sicht erkennt man nunmehr zwei Bestandteile:

1) Banktypologie

2) Geografische Bezeichnung (territoriale Zuständigkeit und Aktivitätsradius der Bank)

Es ist nicht zu übersehen, dass die neuen Bankennamen vollständig säkularisiert sind. 
Die Fülle des sprachlichen Materials (Hagionyme, Adjektive) aus der Domäne der christlichen Religion ist nicht mehr zu finden. Es sei denn, das toponymische Bildungselement des Bankennamens enthält seinerseits ein Hagionym, was im katholischen Italien sehr oft der Fall sein kann:

Banca S. Daniele del Friuli

Banca S. Paolo Brescia

Banca S. Vito di S. Vito al Tagliamento

Banco di S. Geminiano

Cassa di Risparmio di S.Elpidio

Casse Rurale e Artigiana di S. Agata di Montemaggiore Belsito

Casse Rurale e Artigiana di S. Andrea di Castelfranco Veneto

Casse Rurale e Artigiana di S. Anna di Sciara

Casse Rurale e Artigiana di S. Apollinare di Rovigo

Casse Rurale e Artigiana di S. Bernardino di Crema

Casse Rurale e Artigiana di S. Biagio di Fossalta di Portogruaro

Sogar die Bezeichnung "Monte di Pietà" wird im 20. Jahrhundert als obsolet empfunden. Die faschistische Regierung Italiens ändert im Jahr 1938 per Gesetz den Namen dieser Pfandleihen in „Monte di Creditosu Pegno“.

Die weitere Entwicklung im 20. Jahrhundert, vor allem unter dem Einfluss der Auswirkung der neuenBankgesetze 1992 und 1994, bringt die Transformation der italienischen Bankenvon provinziellen lokalen Playern zu großen europäischen Kreditinstituten. Auf der Ebene der Sprache der Bankennamen stellt sich heraus, dass beim Prozess der „Europäisierung" die italienischenBanken Benennungsmuster der großen erfolgreichen Kreditinstitute der Schweiz oder Deutschlandsnachzuahmen versuchen:

Cassa di Risparmio di Modena+Banca del Monte di BolognaàCarimonte Holding

Banche Popolari Unite+Banca Lombarda e PiemonteseàUnione Banche Italiane (UBI Banca) nach dem Muster UBS (Unione Banche Svizzere)

Banca Cattolica Vicentina+ Banco di San Bassiano di Bassano del GrappaàBanca Cattolica del Veneto

UniCredito Italiano+Capitalia àUniCredit Group

Wie man aus den Beispielen deutlich sehen kann, verschwinden nach und nach im Zuge der Umbenennungen aus den italienischen Bankennamen auch die vielen typischen italienischen Toponyme und es etablieren sich immer mehr akronymische, semantisch opake Strukturen oder gar Anglizismen wie bei der Benennenung Hello Bank.

\section{Schlussüberlegungen}

Die Veränderungsprozesse in der Benennung von Banken in Italien zeigen in ihrer Gesamtheit einen existierenden Zusammenhang mit der Stellung der/zur Religion in der italienischen Gesellschaft.

Bei dem Versuch, über ein halbes Jahrtausend einen rotenFaden des Verhältnisses 
zwischen Religion, Kultur, Gesellschaft und Benennung von Banken zu finden, stellt sich heraus, dassOrganisationsnamen, als von Menschen bewusstkreierte Benennungen für von Menschen geschaffene Einrichtungen, in ihrer morphologischen und semantischen Struktur in einer komplexen und dialektischen Art die Entwicklung der Kultur und der Wirtschaftsgeschichte widerspiegeln.

In diesem Sinne können Bankennamen in Italien als sprachliche Artefakte, die den Kultur- und Wertewandel der italienischen Gesellschaft verkörpern und dokumentieren, gewertet werden.

\section{Literaturverzeichnis}

Brendler, A., und S. Brendler (eds.). 2004. Namenarten und ihre Erforschung. Hamburg: Baar.

De Simone, E. 2011. Moneta e banche attraverso i secoli. Milano: De Angeli.

Dräger, K., F. Fahlbusch, D. Nübling (eds.). 2016. Heiligenverehrung und Namengebung. Berlin, New York: De Gruyter Mouton.

Fahlbusch, F. 2017. Unternehmensnamen. Entwicklung-Gestaltung-Wirkung-Verwendung. Berlin: Frank\&Timme

Fischer, F. 2008. Mergers \& Acquisitions und Unternehmensnamen. Onoma 43: 331-350.

Fischer, F. 2015. Von der unsichtbaren Hand Adam Smiths bis zur heutigen Markt-Gläubigkeit der Neoliberalen. Spuren der Religion in der Sprache der Wirtschaft. In Religion \& Sprache, M. Calderon und G. Marko (eds.), 39-50. Frankfurt a. Main: Peter Lang.

Fischer, F. 2017. Organisationsnamen. In Handbook of Business Communikation, G. Mautner und F. Rainer (eds.), 544-550. Berlin, New York: De Gruyter Mouton.

Fischer, F. und H. Wochele. 2012. Identità nazionale e identità europea. Il nome d'impresa come strumento di pubblicità e posizionamento sul mercato: il settore bancario. In Il linguaggio della pubblicità italiano e tedesco: teoria e prassi, P. Cotticelli Kurras und E. RonnebergerSibold (eds.), 105-136. Alessandria: Edizioni Dell'Orso.

Gabler-Wirtschaftslexikon http://wirtschaftslexikon.gabler.de

Gloor, B. 2003. Geld und Geist. In Banks \& Brands, M. Pedergnana, M. Schneider, S. Vogler (eds.), 215-239. Bern: Haupt.

Greule, A. 2016. Heiligennamen in der volkssprachlichen Liturgie. In Heiligenverehrung und Namengebung, K. Dräger, F. Fahlbusch, D. Nübling (eds.), 293-302. Berlin, New York: De Gruyter Mouton.

Gugnoni, G. 2010. Dai Monti di Pietà e le Casse di Risparmio alle Fondazioni Bancarie: evoluzione e profili di riforma. $\mathrm{PhD}$ diss. Università di Bologna.

Koß, G. 2002. Namenforschung. Eine Einführung in die Onomastik. 3rd. ed. Tübingen: Niemeyer.

Kremer, L. 2012. Von der Vereinsbank in Hamburg zu HVB: zur Diachronie deutscher Unternehmensnamen. In Onomastics goes Business. Role and Reliance of Brand. Company and Other Names in Economic Contexts, H. Wochele, J. Kuhn, M. Stegu (eds.), 129-140. Berlin: Logos.

Krüger, D. 2004. Textlinguistische Methoden der Namenforschung. In Namenarten und ihre Erforschung. Ein Lehrbuch für das Studium der Onomastik, A. Brendler und S. Brendler (eds.), 123-152. Hamburg: Baar.

Lopez, R.S., G. Le Goff, Th. W. Blomquist et alii. 1982. L'alba della banca. Le origini del sistema bancario tra Medioevo ed Età Moderna. Bologna: Dedalo. 
Maier, M. 1999. Markenmanagement bei K.reditinstituten. München: Verlag der Förderungsgesellschaft Marketing.

Muzzarelli, M. G. 2001. Il denaro e la salvezza. L'invenzione del Monte di Pietà. Bologna: il Mulino.

Nübling, D., F. Fahlbusch, R. Heuser. 2012. Namen. Eine Einführung in die Onomastik. Tübingen: Narr.

Todeschini, G. 1989. La ricchezza degli ebrei: merci e denaro nella riflessione ebraica e nella definizione cristiana dell'usura alla fine del Medioevo. Bologna: CISAM.

Todeschini, G. 2002. I mercanti e il tempio: la società cristiana e il circolo virtuoso della ricchezza fra Medioevo ed età moderna. Bologna: Il Mulino.

Todeschini, G. 2016. La banca e il ghetto. Bari: Laterza.

Vasil'eva, N. 2004. Institutionennamen. In Namenarten und ihre Erforschung, A. Brendler und S. Brendler (eds.), 605-621. Hamburg: Baar. 